\title{
RIB production and related experiments at EXOTIC
}

\author{
Marco Mazzocco ${ }^{1,2, \star}$ \\ ${ }^{1}$ Dipartimento di Fisica e Astronomia, Universita di Padova, via F. Marzolo 8, I-35131 Padova, Italy \\ ${ }^{2}$ INFN - Sezione di Padova, via F. Marzolo 8, I-35131 Padova, Italy
}

\begin{abstract}
Radioactive nuclei have a very deep relevance in many astrophysical scenarios, from the Big Bang nucleo-synthesis to supernova explosions. Several Nuclear Physics laboratories around the world have been constructing large-scale facilities for the production of Radioactive Ion Beams (RIBs). The main production techniques, i.e. In-Flight and Isotope Separation On Line, which will be reviewed in this contribution. In particular, we will concentrate on the production of light weakly-bound RIBs at the facility EXOTIC, located at INFN-LNL (Italy) and we will describe the most recent experiments.
\end{abstract}

\section{Introduction}

What is the most beautiful painting you have ever seen in your life? If this question is asked to a Nuclear Physicist, the answer might be a little surprising for a standard pedestrian. In fact, there is a rather high probability that the answer could be "The Chart of The Nuclides", i.e. the pictorial representation of the isotopes which was (originally) introduced in the mid-Thirties by the Italian Giorgio Fea [1]. In this chart, each known isotopes occupies a squared box and all boxes are properly ordered according to the atomic number (in the vertical axis) and to the number of neutrons (in the horizontal axis). Each box contains basic information on the nuclear properties of the relative isotope, such as, for instance, natural abundance (if stable) or mean half-live (if radioactive), presence of metastable states, cross section for the bombardment of thermal neutrons and so on. The chart is generally color-coded according to the main (radioactive) decay mode. Black is reserved for stable isotopes, blue for $\beta^{-}$decays, red for $\beta^{+}$decays and electron captures, yellow for $\alpha$ radioactivity, green for spontaneous fission, orange for proton decay and light blue for neutron emission.

A quick look at the nuclide chart immediately gives rise to a first crucial question: what are the limits of existence of nuclei? The search for the heaviest particle-stable configuration of nucleons triggered the long-standing quest for the super-heavies [2]. On the other side, it is also extremely interesting to probe the limits of the nuclear stability along isobaric chains, i.e. let us fix the mass number and let us try to change one-by-one the number of protons and neutrons until we reach on both sides the last combinations stable against particle emission. These two extreme regions of the nuclide chart are called drip-lines and we have the proton- and neutron-drip line in the proton-rich and proton-deficient side the chart, respectively. Among these two lines and within the region of superheavy nuclei, there is a "jungle" of 3532 isotopes [3] observed until the end of 2017, which is a sort of (radioactive) paradise for nuclear physicists and nuclear astrophysicists. However, our present-day

\footnotetext{
^e-mail: marco.mazzocco@pd.infn.it
} 
knowledge of radioactive nuclei is still limited fo about 50-60\% of the nuclei of possible existence. In fact, while the proton drip line has been essentially mapped up to Gold $(Z=79)$, the neutron-rich side is mostly an uncharted territory and this is region where, for instance, the path of the astrophysical rapid neutron capture process ( $r$-process) is mostly located [4].

The interest in the properties of nuclei far from the valley of stability boosted the design and construction of large-scale Radioactive Ion Beams (RIB) facilities, such as for instance the RIBF at RIKEN (Japan) [5], ISOLDE at CERN (Switzerland) [6], ISAC at TRIUMF (Canada) [7], FRS at GSI (Germany) [8] and SPIRAL at GANIL (France) [9]. Besides these sites, there are also several smallscale facilities around the world and a few major RIB facilities under construction, such as FAIR at Darmstadt (Germany) [10], SPIRAL2 at GANIL (France) [11], SPES at INFN-LNL (Italy) [12] and FRIB at MSU (USA) [3].

In this contribution we will at first present the mostly used RIB production mechanisms, then we will discuss the two main RIB production techniques, In-Flight and Isotope Separation On Line (ISOL). Afterwards, we will describe the facility EXOTIC located at INFN-LNL (Italy) and, finally, summarize some of the most recent results obtained by means of this facility.

\section{RIB production mechanisms}

When a facility for the production of Radioactive Ion Bems is laid out, the following guidelines should be taken into account:

- the global production scheme should be fast, as the produced nuclear species are, in most cases, very short-lived and the number of radio-nuclides of interest decaying prior being investigated should be as small as possible;

- the production method should ensure the largest possible production cross section and this can be achieved by optimizing the projectile-target combination, the bombarding energy, the primary beam intensity, the power dissipation into the target, and so on;

- the production should efficient, as we are quite often dealing with very marginal production rates;

- the production should also be quite selective, since the contaminant species could be even several orders of magnitude more abundant than the nuclear species of interest.

There exist four main nuclear reaction mechanisms which can be employed for the production of RIBs, namely: fragmentation, fission, fusion and direct processes.

Fragmentation reactions are peripheral interactions leading to the removal of a few nucleons close to the nuclear surface. To be efficient the colliding energy should be larger than 40-50 MeV/u. In case the fragmentation is induced by an inverse kinematics reaction, i.e. an heavier projectile impinging on a lighter target, the residual nuclei are emerging from the interaction region with "small" recoils and are focused in narrow cones around the primary beam direction. Increasing the beam energy, i.e. building a more powerful driver accelerator, does not help enhancing the fragmentation cross reaction, it rather narrow the opening of the emission cones for the residues, thus diminishing the cost of the spectrometer to be used for the isotope selection. This technique is not extremely selective, can be employed to produce either proton-rich or neutron-rich side of the valley of stability and the projectile to be fragmented can, to some degrees of freedom, be tuned according to region of radioactive nuclei we are interested in.

The production of RIBs by means of the nuclear fission starts, of course, from the use either as projectile or target of a fissile material, typically ${ }^{238} \mathrm{U}$. This process is mostly suitable for the production of neutron-rich nuclei, as it is quite understandable considering the extreme neutron-to-proton 
ratio of radio-isotopes unstable versus spontaneous fission. Fission fragments are emitted with energies (in the center-of-mass reference frame) of about $1 \mathrm{MeV} / \mathrm{u}$ and, in case of fissile projectiles, their kinematic cones around the primary beam direction have openings significantly larger than radioactive nuclei produced via fragmentation. In this case, building a more powerful accelerator can help achieving a much better overall production rate for very exotic fission fragments.

Nuclear fusion could be employed for the production of proton-rich nuclei. In this case, in fact, the compound nucleus is heavier than both projectile and target and, consequently, it will be characterized by a proton-to-neutron ratio much larger than stable isotopes of the same element. Recoil separators exploiting the substantial velocity difference between projectile and evaporation residues can be used to select the radioactive species produced. However, the separation efficiency is often quite limited, since the fusion process has the highest cross section at energies up to a few $\mathrm{MeV} / \mathrm{u}$ and the residues are produced with broad angular, velocity and charge state distributions. So far, nuclear fusion has been used for spectroscopy studies, for the synthesis of super-heavy nuclei, but not for the production of RIBs.

Direct processes are generally employed for the production of light RIBs quite close to the valley of stability. Inverse $(\mathrm{p}, \mathrm{n}),(\mathrm{d}, \mathrm{n})$, or $\left({ }^{3} \mathrm{He}, \mathrm{n}\right)$ reactions, preferably with negative Q-value, are chosen because of the narrow opening angles $\left(\theta_{l a b} \leq 10^{\circ}\right)$ of the reaction products in the laboratory frame. Hydrogen and helium would be the preferred target materials. Unfortunately, there are no He-compounds and H-rich compounds (e.g., $\mathrm{CH}_{2}$ ) cannot withstand the thermal stress induced by an intense primary beam intensity. To circumvent these problems, the use of gas targets with metal windows was undertaken.

\section{RIB production techniques: In Flight vs. ISOL}

In Flight separation and ISOL are considered complementary techniques for the production of RIBs.

In the In Flight production, an energetic heavy ion beam impinges on a thin light target. The target thickness is a crucial parameter, as the reaction products should retain most the projectile initial velocity (typically 90\%) and the deflection introduced by the interaction with the target atoms should be as small as possible. The ions of interest are then selected by a proper combination of electromagnetic fields and atomic interaction. All the procedure is rather fast, as the radioactive ions are already at disposal of the experimentalists right after the transit time through the separator, at most after a few $\mu$ s. Therefore, this technique is particularly suitable for short-lived RIBs. Moreover, since the RIB under production is very energetic, there is no need to equip the facility with a quite expensive post-accelerator. The drawbacks of this method are mostly related to poor optical properties of the outcoming RIBs, in terms of longitudinal and transverse emittance and beam spot on target, and to the limited flexibility on the secondary beam energy, strongly dependent on the energy of the primary beam.

On the other side, the ISOL technique is generally characterized by a light projectile beam, such as for instance a proton or a deuteron beam, impinging on a heavy target. The target station has a certain thickness, in order to stop completely the primary beam, and has to be kept at high temperature. In fact, the radioactive nuclei produced inside the target are thermalized in a catcher, then they are extracted, ionized, mass selected and eventually re-accelerated. Of course, all these different operations, especially those occurring inside the target/catcher container, critically depend on the chemical properties of the species under production and the extraction procedure, for instance, can require a time interval ranging from a few hundreds ms to some tens of seconds. Clearly, very short-lived nuclei are hardly accessible by means of the ISOL technique. However, the possibility of re-accelerating the secondary beam gives the opportunity of having an easy variable beam energy and also a high optical quality, i.e. very small beam emittance. 
In the worldwide scenario, RIBF [5], FRS [8], FAIR [10]and FRIB [3] are In-Flight facilities, whereas ISOLDE [6], ISAC [7], SPIRAL (France) [9], SPIRAL2 [11] and SPES [12] are using (or will use) the ISOL technique.

\section{The Facility EXOTIC at INFN-LNL}

Our project for the design of a facility for the in-flight production of light weakly-bound RIBs at INFN-LNL started in 2002 [14]. We employ heavy ion beams, delivered by the 15 MV XTU-Tandem accelerator, impinging on a gas target which can be filled with light gases, such as ${ }^{1,2} \mathrm{H}$ and ${ }^{3,4} \mathrm{He}$. The commissioning of facility was performed in 2004 [15-17] and a quite substantial upgrade process was held in 2012 [18]. The ion-optical lay-out of the beam-line consists of 3 quadrupole magnets, 1 $30^{\circ}$-dipole magnet, a 1-m long Wien filter and finally a second quadrupole triplet. So far, seven RIBs have been delivered: ${ }^{17} \mathrm{~F},{ }^{8} \mathrm{~B},{ }^{7} \mathrm{Be},{ }^{15} \mathrm{O},{ }^{8} \mathrm{Li}$ and ${ }^{10,11} \mathrm{C}$. In most cases, intensities of to a few units $10^{5}$ pps and purities as good as $99 \%$ were achieved, except for ${ }^{10} \mathrm{C}$ (about $5 \times 10^{3}$ pps) and ${ }^{8} \mathrm{~B}$, where we never exceeded an purity of about $40 \%$ and we hardly reached a intensity of $10^{3}$ pps. The facility is equipped with an event-by-event tracking system based on two $\mathrm{x}-\mathrm{y}$ sensitive high-transparency Parallel Plate Avalanche Counters (PPACs) and an array of $8 \Delta E-E$ telescopes of large-area Double Sided Silicon Strip Detectors (DSSSDs) is located [19,20] at the final focal plane for energy and position measurement and for particle identification purposes.

These RIBs were used for reaction dynamics studies at Coulomb barrier energies for the systems ${ }^{17} \mathrm{~F}+{ }^{208} \mathrm{~Pb}[21],{ }^{17} \mathrm{~F}+{ }^{58} \mathrm{Ni}[22],{ }^{17} \mathrm{~F}+{ }^{1} \mathrm{H}[23],{ }^{8} \mathrm{~B}+{ }^{28} \mathrm{Si}[24],{ }^{7} \mathrm{Be}+{ }^{58} \mathrm{Ni}[25],{ }^{8} \mathrm{Li}+{ }^{90} \mathrm{Zr}[26,27]$ and ${ }^{7} \mathrm{Be}+{ }^{28} \mathrm{Si}[28,29]$. More recently, a resonant scattering experiment has been performed for the system ${ }^{15} \mathrm{O}+{ }^{4} \mathrm{He}[30]$ and also the capabilities of the facility to be employed as a separator for heavy-ion fusion reaction products were tested [31].

In November 2015 a first experiment of astrophysical relevance was performed. The goal of the measurement was the investigation of the reaction ${ }^{7} \mathrm{Be}(\mathrm{n}, \alpha)^{4} \mathrm{He}$ by means of the Trojan Horse Method $[32,33]$ and the surrogate reaction ${ }^{7} \mathrm{Be}(\mathrm{d}, \alpha \mathrm{p}){ }^{4} \mathrm{He}$. This reaction is the second most dominant ${ }^{7} \mathrm{Be}$ destruction mechanism in the Big-Bang Nucleosynthesis (BBN) model and might provide a nuclear physics explanation for the ${ }^{7} \mathrm{Li}$ cosmological problem, i.e. the experimental evidence that in metalpoor stars the ${ }^{7} \mathrm{Li}$ abundance is about a factor of 3 smaller with respect to standard BBN theoretical predictions [34]. The data analysis is currently in progress and very promising results have already been communicated to the International Conference Nuclear Physics in Astrophysics VIII (NPA 8, Catania June 2017) [35].

\section{Summary and Perspectives}

The continuously growing availability of RIBs will make possible the study on earth of nuclear reactions typically occurring only in explosive astrophysical scenarios. The construction of new facilities and the complementarity of the two main production techniques, In-Flight and ISOL, will even widen our present perspectives. Within this framework, a first generation RIB facility was built at INFNLNL and, after an initial phase mainly devoted to reaction dynamics studies at near-barrier energies, a new program aimed at investigating reactions of astrophysical relevance has recently started.

\section{References}

[1] G. Fea, Il Nuovo Cimento 12, 368 (1935).

[2] Yu.Ts. Oganessian and V.K. Utyonkov, Rep. Prog. Phys. 78, 03601 (2015). 
[3] https://people.nscl.msu.edu/ thoennes/isotopes/

[4] M.S. Smith and K.E. Rehm, Annu. Rev. Nucl. Part. Sci. 51, 91 (2001).

[5] http://www.nishina.riken.jp/RIBF/

[6] http://isolde.web.cern.ch/

[7] http://www.triumf.ca/research-program/research-facilities/isac-facilities

[8] https://www-win.gsi.de/frs/index.htm

[9] http://pro.ganil-spiral2.eu/laboratory/ganil-accelerators/spiral

[10] http://www.fair-center.eu/

[11] http://pro.ganil-spiral2.eu/spiral2/what-is-spiral2

[12] https://web.infn.it/spes/

[13] https://frib.msu.edu/

[14] V.Z. Maidikov et al., Nucl. Phys. A 746, 389c (2004).

[15] D. Pierroutsakou et al., Eur. Phys. J. Spec. Top. 150, 47 (2007).

[16] F.Farinon et al., Nucl. Instrum.Methods B 266, 4097 (2008).

[17] M. Mazzocco et al., Nucl. Instrum.Methods B 266, 4665 (2008).

[18] M. Mazzocco et al., Nucl. Instrum.Methods B 317, 223 (2013).

[19] E. Strano et al., Nucl. Instrum. Methods B 317, 657 (2013).

[20] D. Pierroutsakou et al., Nucl. Instrum. Methods A 834, 46 (2016).

[21] C. Signorini et al., Eur. Phys. J. A 44, 63 (2010)

[22] M. Mazzocco et al., Phys. Rev. C 82, 054604 (2010)

[23] N. Patronis et al., Phys. Rev. C 85, 024609 (2012)

[24] A. Pakou et al., Phys. Rev. C 87, 014619 (2013)

[25] M. Mazzocco et al., Phys. Rev. C 92, 024615 (2015)

[26] A. Pakou at al., Eur. Phys. J. A 51, 55 (2015)

[27] A. Pakou at al., Eur. Phys. J. A 51, 90 (2015)

[28] O. Sgouros et al., , Phys. Rev. C 94, 044623 (2016)

[29] O. Sgouros et al., , Phys. Rev. C 95, 054609 (2017)

[30] D. Torresi et al., Phys. Rev. C 96, 044317 (2017)

[31] E. Strano et al., Nucl. Instrum. Methods A 877, 293 (2018)

[32] C. Spitaleri et al., Physics of At. Nuclei 74, 1725 (2011)

[33] R.E. Tribble et al., Rep. Prog. Phys. 77, 106901 (2014)

[34] C. Broggini et al., Jour. Cosm. Astrop. Phys. 6, 30 (2012)

[35] L. Lamia et al., EPJ Web of Conferences 165, 01032 (2017) 